-Original-

\title{
Insulin Resistance, Steatohepatitis, and Hepatocellular Carcinoma in a New Congenic Strain of Fatty Liver Shionogi (FLS) Mice with the Lep ${ }^{o b}$ Gene
}

\author{
Masahiko SOGA $^{1)}$, Setsuko HASHIMOTO ${ }^{2)}$, Yoshio KISHIMOTO ${ }^{3)}$, \\ Tsutomu HIRASAWA ${ }^{2)}$, Susumu MAKINO $^{3,5)}$, and Shuichiro INAGAKI ${ }^{4)}$
}

\begin{abstract}
${ }^{1)}$ Discovery Research Laboratories, Shionogi \& Co., Ltd., 3-1-1 Futaba-cho, Toyonaka, Osaka 561-0825,
${ }^{2)}$ Developmental Research Laboratories and ${ }^{3)}$ Aburahi Laboratories, Shionogi \& Co., Ltd., 1405 Gotanda, Koka-cho, Koka, Shiga 520-3423, ${ }^{4}$ Discovery Research Laboratories, Shionogi \& Co., Ltd., 5-12-4 Sagisu, Fukushima-ku, Osaka 553-0002, and ${ }^{5)}$ KAC Co., Ltd., 91 Higashisaka, Ritto, Shiga 520-3001, Japan
\end{abstract}

\begin{abstract}
In order to examine the influence of obesity on metabolic disorder and liver pathogenesis of the Fatty Liver Shionogi (FLS) mouse, which develops hereditary fatty liver and spontaneous liver tumors, we established a new congenic strain named FLS-Lep ${ }^{o b}$. The $L e p^{o b}$ gene of the C57BL/6JWakShi (B6)-Lep ${ }^{o b} / L e p^{o b}$ mouse was transferred into the genome of the FLS mouse, by backcross mating. FLS-Le $p^{o b} / L e p^{o b}$ mice were maintained by intercrossing between $L e p^{o b}$-heterozygous littermates. The FLS-Lep $p^{o b} / L e p^{o b}$ mice of both sexes developed remarkable hyperphagia, obesity and type 2 diabetes mellitus. At 12 weeks of age, glucosuria was detected in all male and female FLS-Lep $p^{o b} / L e p^{a b}$ mice. Biochemical examination demonstrated that the FLS-Lep ${ }^{o b} / L e p^{o b}$ mice have severe hyperlipidemia and hyperinsulinemia. The livers of FLS-Lep ${ }^{o b} / L_{e}{ }^{a b}$ mice showed microvesicular steatosis and deposition of large lipid droplets in hepatocytes throughout the lobules. The steatohepatitis-like lesions including the multifocal mononuclear cell infiltration and clusters of foamy cells were observed earlier in FLS-Lep ${ }^{o b} / L_{e} p^{o b}$ mice than in FLS mice. B6-Lep $p^{o b} / L e p^{o b}$ mice did not show hepatic inflammatory change. Furthermore, FLS-Lep ${ }^{o b} / L e p^{o b}$ mice developed multiple hepatic tumors including hepatocellular adenomas and carcinomas following steatohepatitis. In conclusion, the FLS-Lep $p^{o b} / L e p^{o b}$ mice developed steatohepatitis and hepatic tumors following hepatic steatosis. The FLS-Lep ${ }^{o b} / L_{e} p^{a b}$ mouse with obesity and type 2 diabetes mellitus might be a useful animal model for human non-alcoholic steatohepatitis (NASH).
\end{abstract}

Key words: FLS-Lep ${ }^{o b} / L e p^{o b}$ mouse, hepatocellular carcinoma, insulin resistance, nonalcoholic steatohepsatitis, obesity

\section{Introduction}

Fatty liver is defined as abnormal accumulation of triglyceride within hepatocytes. In humans, fatty liver is a common hepatic reaction to chronic alcohol ingestion, and is often seen in persons with obesity, type 2 diabetes mellitus, and hyperlipidemia. It is well known that chronic alcohol intake is a risk factor of severe he- 
patic disorders including steatohepatitis, cirrhosis, and carcinogenesis. On the other hand, non-alcoholic fatty liver has not attracted much attention until recently because the condition was thought to be a reversible benign disease. In 1980, Ludwig et al. reported that alcoholic steatohepatitis-like lesions were observed in persons who did not consume alcohol, and that form of steatohepatitis was named non-alcoholic steatohepatitis (NASH) [17]. NASH is microscopically characterized by hepatic steatosis, lobular inflammation, ballooning degeneration of hepatocytes, Mallory bodies, and pericellular fibrosis [18]. Furthermore, recent studies have suggested that NASH may progress to fibrosis, cirrhosis, and hepatocellular carcinoma $[2,9,25,34]$. The prevalence of NASH is $2.1-6.3 \%$ in the general populations, and in many patients, NASH is associated with obesity, diabetes mellitus, and hyperlipidemia [3, 22, 27]. NASH has become an important health problem with the increase of obesity and type 2 diabetes mellitus in developed countries.

Previously, we reported the establishment of the Fatty Liver Shionogi (FLS) mouse [30]. This strain of mouse shows lipid deposition in hepatocytes from the neonatal stage, and the degree of liver lipid (mainly triglyceride) accumulation increases as the mouse grows without obesity. In addition to lipid deposition, steatohepatitis-like lesions with micro-abscesses and infiltration of mononuclear cells is observed in FLS mice. A preliminary genetic cross study suggested that the fatty liver formation in the FLS mice is caused by a complex polygenic trait. Interestingly, aged FLS mice frequently develop hepatocellular adenoma, carcinoma, and peliosis hepatis [31]. Therefore, the FLS mouse might be a useful animal model for human NASH.

$\mathrm{C} 57 \mathrm{BL} / 6 \mathrm{~J}-\mathrm{Lep}^{o b} / \mathrm{Lep}^{o b}$ mice are often used as a model animal for human obesity and type 2 diabetes mellitus. The mutation of the $L e p^{o b}$ gene coding the leptin protein causes remarkable hyperphagia and obesity [35]. Leptin, which is secreted from white adipose tissue with the increase of the body fat, suppresses of feeding behavior and increases energy consumption through leptin receptors in the hypothalamus [7].

In this study, we attempted to transfer the $L e p^{o b}$ gene of the C57BL/6JWakShi (B6)-Lep ${ }^{o b} / L e p^{o b}$ mouse into the FLS mouse to examine the influence of obesity on meta- bolic disorder and liver pathogenesis in the FLS mouse. A new congenic strain of mouse, FLS-Lep ${ }^{o b} / L e p^{o b}$, was established by backcross mating of FLS and B6-Lep ${ }^{o b} /+$ mice. The congenic strain developed remarkable obesity and diabetic symptoms, and its hepatic steatosis and steatohepatitis developed quicker than those of FLS and B6- $L e p^{o b} / L e p^{o b}$ mice. Furthermore, FLS-Lep ${ }^{o b} / L e p^{o b}$ mice developed hepatocellular carcinoma earlier than FLS mice. Here we describe the features of the obese-diabetic symptoms, steatohepatitis and hepatic tumors observed in the new congenic strain with the $L e p^{o b}$ mutation.

\section{Materials and Methods}

\section{Animals}

FLS mice were established and have been maintained at our laboratory [30]. B6-Lep $p^{o b} / L e p^{o b}$ mice, the donor of the $L e p^{o b}$-gene, were obtained from Wakayama Medical University in 1974, and have been maintained at our laboratory by mating of the $L e p^{o b}$ heterozygotes. C57BL/6JShi mice were used in this study as a control lean mouse. All mice were kept in our specific pathogenfree facilities, which are free of the following microorganisms: HVJ, MHV, Bordetella bronchiseptica, Corynebacterium kutscheri, Mycoplasma pulmonis, Tyzzer's organism (Clostridium piliforme), Pasteurella pneumotropica, Salmonella sp., Pseudomonas aeruginosa, Staphylococus aureus, and Helicobacter hepaticus. The inside of the animal facilities was maintained at constant temperature $\left(23 \pm 1{ }^{\circ} \mathrm{C}\right)$ and humidity $(60 \pm 5 \%)$ with lighting for $12 \mathrm{~h}$ daily (light from 06:00 to 18:00). Mice were housed in polycarbonate cages with hardwood chip bedding. Commercial chow diet (CA-1; Clea Japan Inc., Tokyo, Japan) containing crude protein, $27 \%$, and crude fat, $4.6 \%$, and drinking tap water were available $a d l i$ bitum. Chow was autoclaved at $121^{\circ} \mathrm{C}$ for $7 \mathrm{~min}$, and drinking water was sterilized by filtration and UV light. All experiments in this study followed the Guidelines for Animal Experimentation of the Japanese Association for Laboratory Animal Science [15].

\section{Clinical characterizations}

Body weights of mice used in this study were recorded until 60 weeks of age from 5 weeks of age. The appearance of glucosuria was monitored using Testape ${ }^{\circledR}$ 
(Shionogi \& Co., Ltd., Osaka, Japan) until 60 weeks of age from 3 weeks of age. The mice showing Testape ${ }^{\circledR}$ values of $1+(>100 \mathrm{mg} / \mathrm{dl}$ urine $)$ or higher were diagnosed as having overt diabetes. Food consumption, water intake, and excreted urine volume were measured at 10 and 20 weeks of age, by placing the mice in stainless metabolic cages.

\section{Plasma biochemical analysis}

Mice were anesthetized by intraperitoneal administration of Nembutal ${ }^{\circledR}$ (Abbott Laboratories, North Chicago, IL, USA) under the non-fasting condition at 10 weeks of age. Blood samples were collected in a heparinized syringe (between 09:00 and 10:00) from the heart. The plasma was immediately separated by centrifugation (10 $\min , 1,500 \times g$ ). The plasma triglyceride level was measured by an enzymatic method using a commercial kit, TRIGLYZYME-600 ${ }^{\circledR}$ (Eiken Chemical, Tokyo, Japan). Plasma cholesterol and phospholipids were measured by enzymatic methods using Cholesterol E-Test Wako ${ }^{\circledR}$ and Phospholipid C-Test Wako ${ }^{\circledR}$ (Wako Pure Chemical, Osaka, Japan), respectively. Blood glucose was measured by the glucose oxidase method using Glucose BTest Wako ${ }^{\circledR}$ (Wako Pure Chemical). The plasma insulin concentration was determined by enzyme immunoassay (GLAZYME $^{\circledR}$ Insulin EIA-Test; Wako Pure Chemical) with human insulin as a standard. Plasma alanine transaminase (ALT) activity was measured using an automatic analyzer (model 705; Hitachi, Tokyo, Japan) with reagents from Wako Pure Chemical.

\section{Measurement of liver lipids}

Mice were sacrificed under anesthesia induced by administration of an overdose of Nembutal ${ }^{\circledR}$ at 10 weeks of age. Livers were immediately removed, and weighed. Part of each liver was homogenized in distilled water, and the lipids were extracted by shaking in cold chloroform:methanol (v/v, 2:1), as described by Folch et al. [6]. Liver triglyceride, total cholesterol, and phospholipid contents were measured by enzymatic methods using commercial kits (Triglyceride-E, Cholesterol-E, and Phospholipid-C Test Wako ${ }^{\circledR}$; Wako Pure Chemical).

\section{Oral glucose tolerance test (OGTT)}

At 10 and 20 weeks of age, female mice were orally administrated glucose ( $2 \mathrm{~g} / \mathrm{kg}$ body weight) after overnight fasting. Blood samples from the infraorbital venous plexus were obtained consecutively at $0,30,60$, 120, and 180 min after glucose administration, by a heparinized capillary. Plasma glucose level was measured by Glucose B-Test ${ }^{\circledR}$ (Wako Pure Chemical).

\section{Expression of leptin mRNA in adipose tissue}

The mouse leptin oligonucleotide probe (5'-CAG TGA CCC TCT GCT TGG CGG ATA CCG ACT GCG TGT GTG A-3') for northern blot hybridization was established in an upstream region of the mutation point [35]. The probe was labeled with DIG-dUTP using DIG 3'-Tailing Kit (Boehringer Mannheim, Mannheim, Germany). Total RNA was extracted from periuterine white adipose tissue in 10-week-old mice, using RNeasy ${ }^{\circledR}$ Total RNA Kit (QIAGEN, Hilden, Germany). Total RNA (15 $\mu \mathrm{g} /$ lane) was fractionated by formaldehyde-agarose gel electrophoresis. The RNA was then transferred to a charged Nytran ${ }^{\circledR}$ Nylon Membrane (Schleicher \& Schuell, Keene, NH, USA) by capillary blotting, and fixed with UV light. Hybridization was carried out overnight at $65^{\circ} \mathrm{C}$ in hybridization buffer containing the probes $(100 \mathrm{ng} / \mathrm{ml})$. Detection of the signal was carried out using alkline phosphatase-conjugated anti-DIG antibody (Boehringer Mannheim) with CDP-star ${ }^{\mathrm{TM}}$ (Boehringer Mannheim) as a chemiluminescent substrate. The signal was visualized by exposure of membranes to Hyper-film ECL (Amersham, Buckinghamshire, England). A $\beta$-actin DNA probe was used to monitor the amount of total RNA.

\section{Histological examination}

At 10-40 weeks of age, FLS-Lep ${ }^{o b} / L e p^{o b}, \mathrm{~B} 6-L e p^{o b} /$ $L e p^{o b}$, and FLS mice were sacrificed anesthesia induced by administration of Nembutal ${ }^{\circledR}$. The livers were immediately removed and fixed in 10\% phosphate-buffered formalin ( $\mathrm{pH}$ 7.4). They were dehydrated in $100 \%$ ethanol and embedded in paraffin at $58^{\circ} \mathrm{C}$ using standard procedures. Sections ( $5 \mu \mathrm{m}$ thick) were cut and stained with hematoxylin \& eosin (HE) for observation under an optical microscope. Mice over the age of 12 months were macroscopically observed for the development of 
hepatic tumors. The HE sections were prepared as described above.

\section{Statistical analysis}

The data are expressed as mean \pm SE. One-way analysis of variance (ANOVA) followed by Student's $t$-test or by Welch's $t$-test was used for statistical analysis of the data. The criterion for significance was taken as $P<0.05$.

\section{Results}

\section{Establishment of the congenic strain}

First, mating was carried out between male B6-Lep ${ }^{o b} /+$ and female FLS mice to obtain $\mathrm{F}_{1}$ hybrids carrying the $L e p^{o b}$ allele. The $\mathrm{F}_{1}$ mice were test-crossed with B6$L e p^{o b} /+$ mice for selection of $L e p^{o b}$ heterozygous $\mathrm{F}_{1}$ females among the littermates. $\mathrm{F}_{1}$ female $\left(\operatorname{Lep}^{o b} /+\right)$ was backcrossed to male FLS mouse. Subsequently, the backcross mating was done between male progenies $\left(L e p^{o b} /+\right)$ and female FLS mice, and repeated for 12 generations. The new congenic strain was named the FLS-Lep ${ }^{o b} / L e p^{o b}$ mouse (Fig. 1A). Because FLS-Lep ${ }^{o b} /$ $L e p^{o b}$ mice are infertile, $L e p^{o b}$ homozygous mice must be obtained by mating of $L e p^{o b}$ heterozygotes.

The antisense oligonucleotide probe for leptin mRNA detected a single band $(\sim 4.1 \mathrm{~kb})$ in northern bloting of total RNA from periuterine white adipose tissues (Fig. 1B). The leptin mRNA expression in FLS-Lep ${ }^{o b} /$ Lep $^{o b}$ and $\mathrm{B} 6-L e p^{o b} / L e p^{o b}$ mice was higher than in lean mice. The expression of leptin mRNA in FLS mice was slightly higher than in C57BL/6J/Shi mice.

\section{Obesity-related characters}

Temporal changes in body weights of FLS-Lep ${ }^{o b} / \mathrm{Lep}^{o b}$, B6- $L e p^{o b} / L_{e p}^{o b}$, FLS, and C57BL/6Jshi mice are shown in Fig. 2A. The body weights of FLS-Lep ${ }^{o b} / L e p^{o b}$ mice of both sexes were already higher than those of B6$L e p^{o b} / L e p^{o b}$ and FLS mice at 5 weeks of age. Subsequently, the FLS-Lep $p^{o b} / L e p^{o b}$ and B6-Lep $p^{o b} / L e p^{o b}$ mice showed body weight gain until 15 weeks of age. The mean body weight of the FLS-Lep ${ }^{o b} /$ Lep $^{o b}$ mice reached its peak, 58 $\mathrm{g}$ for males at 40 weeks and $59 \mathrm{~g}$ for females, at 35 weeks of age. The body weights of FLS-Lep ${ }^{o b} / L e p^{o b}$ mice of both sexes were higher than those of $\mathrm{B} 6-L e p^{o b} / L e p^{o b}$ mice until

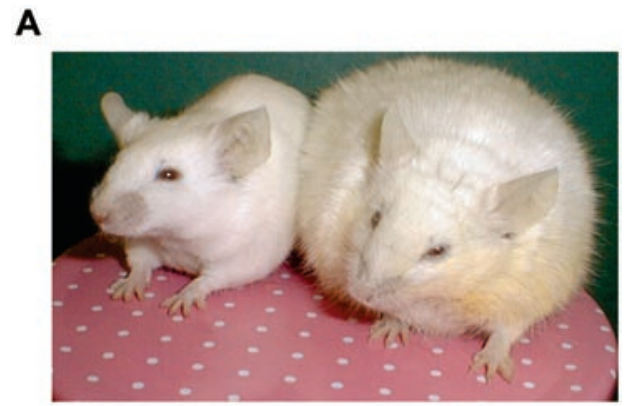

B

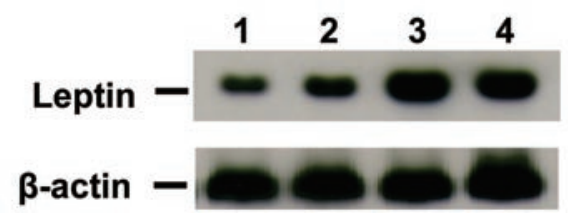

Fig. 1. (A) Appearance of FLS (left) and FLSLep ${ }^{o b} /$ Lep $^{o b}$ mice (right). (B) Leptin mRNA expression in adipose tissue. Northern blot hybridization was performed using probes established in an upstream region of the mutation point. Lane 1: C57BL/6JShi, Lane 2: FLS, Lane 3: C57BL/6JWakShi$L e p^{o b} / L_{e}{ }^{o b}$, Lane 4: FLS-Lep ${ }^{o b} / L_{e p}{ }^{o b}$.

55 weeks of age.

Food consumption of FLS-Lep ${ }^{o b} / L e p^{o b}$ mice of both sexes was increased at 10 weeks compared with B6$L_{e p}^{o b} /$ Lep $^{o b}$ mice (Fig. 2B). At this stage, the food consumption of lean FLS mice was 3-4 g (data not shown). At 20 weeks of age, the food consumption of both these obese mouse strains showed a tendency to decrease from the levels seen at 10 weeks.

\section{Diabetes-related characters}

The incidences of overt diabetes in FLS-Lep $p^{o b} / L^{e p} p^{o b}$ and B6-Lep ${ }^{o b} / L_{e p}^{o b}$ mice are shown in Fig. 3. At 3 weeks of age, $18 \%(5 / 28)$ of male and $12 \%(3 / 25)$ of female FLS-Lep ${ }^{o b} /$ Lep $^{o b}$ mice had already developed overt diabetes. At 12 weeks of age, glucosuria was observed in all male and female FLS-Lep $p^{o b} / L e p^{o b}$ mice. Subsequently, the incidence of diabetes in FLS-Lep ${ }^{o b} /$ Lep $^{o b}$ mice gradually decreased with age, and glycosuria disappeared at 54 weeks of age. On the other hand, diabetes had developed in only 43\% (13/30) of male B6-Lep ${ }^{o b} /$ Lep $^{o b}$ mice at 12 weeks and 23\% (7/30) of females at 6 weeks of age. The 
A
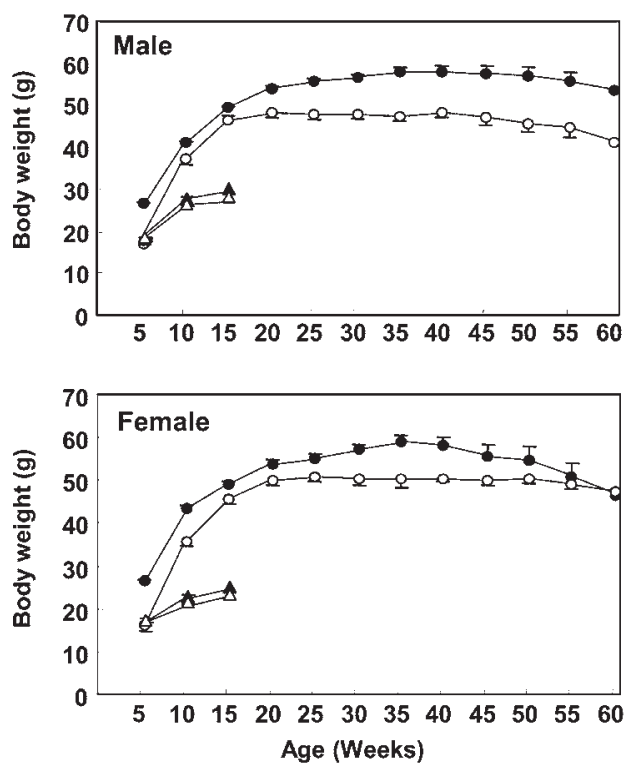
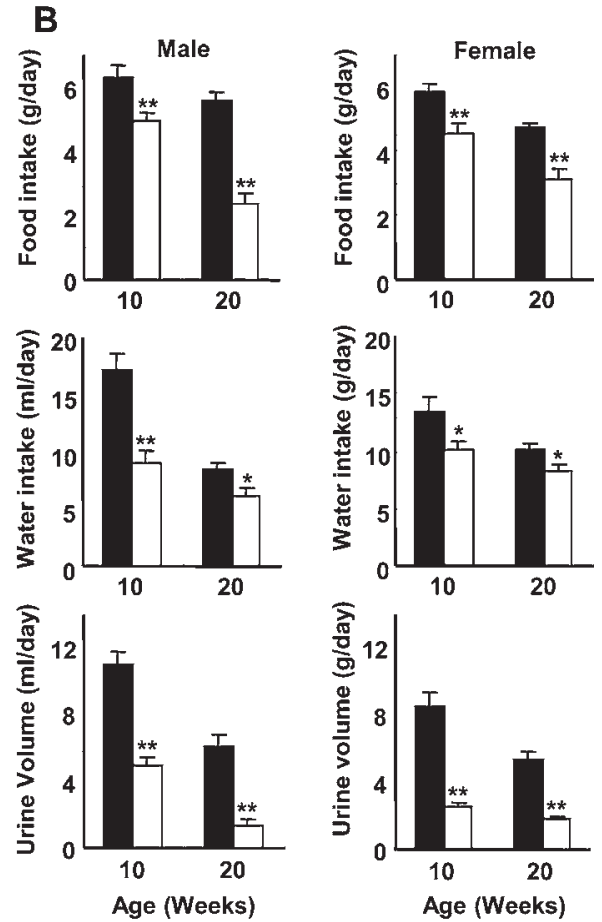

Fig. 2. (A) Growth curves of $L e p^{o b} / L e p^{o b}$ mice. Black circles, FLS-Lep $p^{o b} / L e p^{o b}$ mice (17 males, 18 females). White circles, C57BL/6JWakshi-Lep ${ }^{o b} /$ Lep $^{o b}$ mice (13 males, 19 females). Black triangles, FLS mice (20 males, 18 females). White triangles, C57BL/6J/Shi mice (21 males, 18 females). Data are expressed as the mean \pm SE. (B) Food consumption, water intake, and urine volume of FLS$L e p^{o b} / L e p^{o b}$ and C57BL/6JWakshi-Lep $p^{o b} / L e p^{o b}$ mice of both sexes at 10 and 20 weeks of age. Black columns, FLS-Lep ${ }^{o b} / L_{e p}^{o b}$ mice (6 males, 6 females). White column, C57BL/6JWakShi-Lep $p^{o b}$ / $L e p^{o b}$ mice (6 males, 6 females). ${ }^{*} P<0.05, * * P<0.01$ vs. FLS-Lep ${ }^{o b} / L e p^{o b}$ mice.

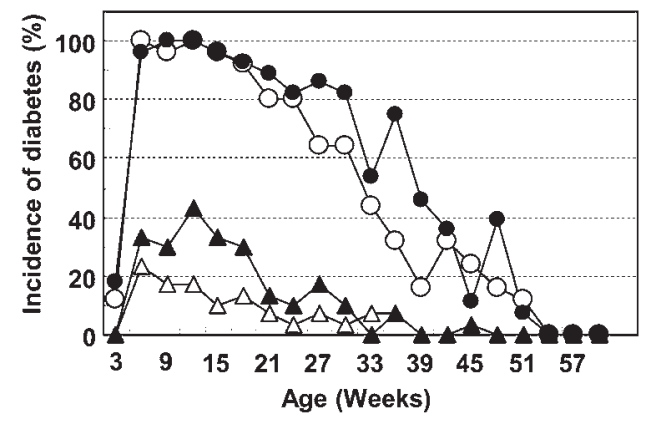

Fig. 3. Incidence of glucosuria in FLS-Lep $p^{o b} / L e p^{o b}$ and C57BL/6JWakshi-Lep $p^{o b} / L e p^{o b}$ mice. Black circles, male FLS-Lep $p^{o b} /$ Lep $^{o b}$ mice $(\mathrm{n}=28)$. White circles, female FLS-Lep ${ }^{o b} /$ Lep ${ }^{o b}$ mice $(\mathrm{n}=25)$. Black triangles, male C57BL/6JWakShi-Lep $p^{o b} /$ Lep $^{o b}$ mice $(\mathrm{n}=30)$. White triangles, female C57BL/6JWakShiLep $^{o b} /$ Lep $^{o b}$ mice $(\mathrm{n}=30)$. The appearance of glucosuria was monitored using Testape ${ }^{\circledR}$. The mice which showed Testape values of $1+(>100 \mathrm{mg} / \mathrm{dl}$ urine $)$ or higher were diagnosed as having overt diabetes.
B6-Lep ${ }^{o b} /$ Lep $^{o b}$ mice also showed a decrease in the incidence of overt diabetes with aging. The FLS and C57BL/6JShi mice did not show glucosuria. Water intake and urine volume of the FLS-Lep ${ }^{o b} /$ Lep $^{o b}$ mice at 10 and 20 weeks of age are shown in Fig. 2B. Polyposia and polydipsia were seen in the FLS-Lep ${ }^{o b} /$ Lep $^{o b}$ mice at 10 weeks when glucosuria peaked. Subsequently, water intake and urine volume of both strains decreased with aging. The results of female mice at 10 and 20 weeks of age in OGTT are shown in Fig. 4. Ten-week-old FLS-Lep ${ }^{o b} /$ $L e p^{o b}$ mice showed severe glucose intolerance and a remarkable elevation of plasma glucose was found after the oral administration which remained at a high level (Fig. 4A) throughout the test period. At the same age, B6$L e p^{o b} / L e p^{o b}$ mice also showed a marked elevation of plasma glucose, but it returned to the initial level at 180 min. The results of FLS mice in OGTT suggested that this strain has mild insulin resistance (Fig. 4A and 4B). 

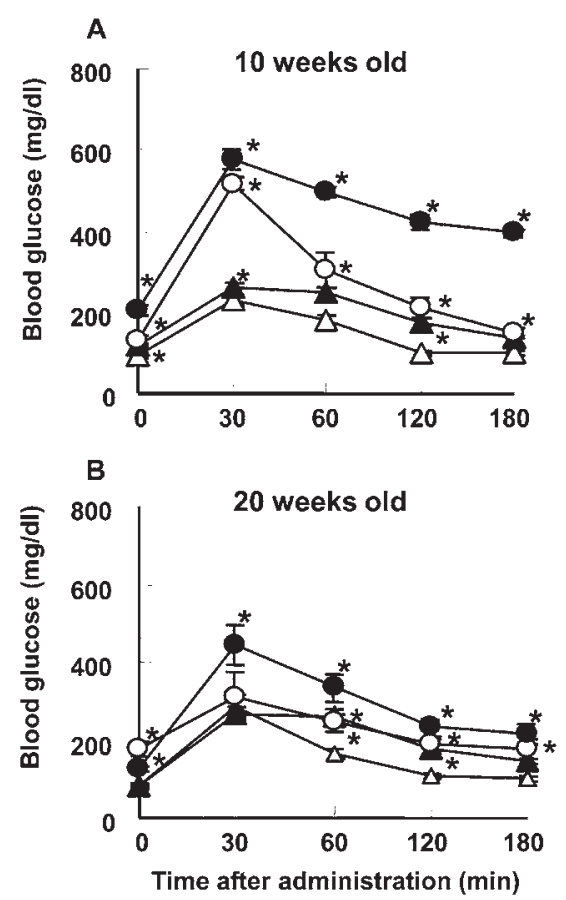

Fig. 4. Oral glucose tolerance test results of female FLS$L e p^{o b} / L e p^{o b}$, C57BL/6JWakShi-Lep ${ }^{o b} / L e p^{o b}$, FLS, and C57BL/6JShi mice at 10 and 20 weeks of age. Black circles, FLS-Lep $p^{o b} / L e p^{o b}$ mice $(\mathrm{n}=5)$. White circles, C57BL/6JWakShi-Lep $p^{o b} /$ Lep $^{o b}$ mice $(\mathrm{n}=5)$. Black triangles, FLS mice $(\mathrm{n}=5)$. White triangles, C57BL/6JShi mice $(\mathrm{n}=5-6) . P<0.05$ vs. C57BL/6JShi mice.

\section{Plasma biochemistry}

Plasma biochemical features of 10-week-old FLS$L e p^{o b} / L e p^{o b}$, B6-Lep $p^{o b} / L e p^{o b}$, FLS, and C57BL/6Jshi mice are summarized in Table 1 . The plasma triglyceride level of male FLS-Lep ${ }^{o b} / L_{e p}{ }^{o b}$ mice was $883 \mathrm{mg} / \mathrm{dl}$ which was significantly higher (2.6-fold) than that of B6-Lep $p^{o b} / L e p^{o b}$ mice, and was also significantly higher (3-fold) than that of FLS mice. In females, the plasma triglyceride level was significantly higher in FLS-Lep ${ }^{o b}$ / $L e p^{o b}$ mice than in B6-Lep ${ }^{o b} / L e p^{o b}$ and lean mice. Plasma cholesterol and phospholipid levels were also significantly higher in male and female FLS-Lep ${ }^{o b} / L e p^{o b}$ mice than in B6-Lep ${ }^{o b} / L e p^{o b}$ and lean mice. Glucose levels of male and female FLS-Lep $p^{o b} / L e p^{o b}$ mice were 572 and $499 \mathrm{mg} / \mathrm{dl}$, respectively, and they were significantly higher than those of B6-Lep ${ }^{o b} / L e p^{o b}$ and lean mice. Plasma insulin levels of male FLS-Lep ${ }^{o b} / L_{e p} p^{o b}$ and B6$L e p^{o b} / L e p^{o b}$ mice were, respectively, 30- and 20-fold higher than that of each control lean mice. Female $L e p^{o b} / L^{o p} p^{o b}$ strains also showed a remarkable increase in plasma insulin. Plasma ALT activities of male FLS$L e p^{o b} / L_{e p}{ }^{o b}$ and B6-Lep ${ }^{o b} / L_{e p}{ }^{o b}$ mice were 7.6- and 2.8fold higher than those of FLS and C57BL/6JShi mice,

Table 1. Plasma biochemical parameters of male and female FLS-Lep ${ }^{o b} / L e p^{o b}$, C57BL/6JWakShi-Lep ${ }^{o b} /$ Lep ${ }^{o b}$, FLS, and C57BL/6JShi mice at 10 weeks of age

Male

\begin{tabular}{lcccc}
\hline & $\begin{array}{c}\text { C57BL/6JShi } \\
(\mathrm{n}=9)\end{array}$ & $\begin{array}{c}\text { FLS } \\
(\mathrm{n}=8)\end{array}$ & $\begin{array}{c}\text { C57BL/6JWakShi-Lep } \\
(\mathrm{n}=10)\end{array}$ & $\begin{array}{c}\text { FLS } / \text { Lep }^{o b} / \text { Lep }^{o b} \\
(\mathrm{n}=8)\end{array}$ \\
\hline Triglyceride $(\mathrm{mg} / \mathrm{dl})$ & $58 \pm 4$ & $295 \pm 29$ & $335 \pm 22^{*}$ & $883 \pm 83^{* \#}$ \\
Cholesterol $(\mathrm{mg} / \mathrm{dl})$ & $87 \pm 3$ & $138 \pm 6$ & $313 \pm 7 *$ & $583 \pm 30^{* \#}$ \\
Phospholipid $(\mathrm{mg} / \mathrm{dl})$ & $156 \pm 5$ & $242 \pm 9$ & $463 \pm 12^{*}$ & $945 \pm 52^{* \#}$ \\
Glucose $(\mathrm{mg} / \mathrm{dl})$ & $171 \pm 8$ & $191 \pm 14$ & $487 \pm 16^{*}$ & $572 \pm 32^{* \#}$ \\
Insulin $(\mu \mathrm{U} / \mathrm{ml})$ & $27 \pm 4$ & $15 \pm 1$ & $553 \pm 7^{*}$ & $464 \pm 21^{* \#}$ \\
ALT $(\mathrm{IU} / \mathrm{l})$ & $50 \pm 2$ & $63 \pm 4$ & $142 \pm 10^{*}$ & $479 \pm 47^{* \#}$ \\
\hline
\end{tabular}

Female

\begin{tabular}{lcccc}
\hline & $\begin{array}{c}\text { C57BL/6JShi } \\
(\mathrm{n}=10)\end{array}$ & $\begin{array}{c}\text { FLS } \\
(\mathrm{n}=9)\end{array}$ & $\begin{array}{c}\text { C57BL/6JWakShi-Lep } \\
(\mathrm{n}=10)\end{array}$ & $\begin{array}{c}\text { FLS } \\
\text { Le } \text { Lep }^{o b} / \text { Lep }^{o b} \\
(\mathrm{n}=8)\end{array}$ \\
\hline Triglyceride (mg/dl) & $23 \pm 4$ & $186 \pm 12$ & $89 \pm 8^{*}$ & $497 \pm 60^{* \#}$ \\
Cholesterol (mg/dl) & $84 \pm 2$ & $118 \pm 5$ & $314 \pm 7 *$ & $552 \pm 32^{* \#}$ \\
Phospholipid (mg/dl) & $137 \pm 4$ & $196 \pm 7$ & $437 \pm 22^{*}$ & $937 \pm 56^{* \#}$ \\
Glucose (mg/dl) & $167 \pm 5$ & $176 \pm 13$ & $408 \pm 24^{*}$ & $499 \pm 32^{* \#}$ \\
Insulin $(\mu \mathrm{U} / \mathrm{ml})$ & $10 \pm 1$ & $15 \pm 1$ & $527 \pm 20^{*}$ & $407 \pm 41^{* \#}$ \\
ALT (IU/l) & $45 \pm 4$ & $41 \pm 2$ & $273 \pm 25^{*}$ & $558 \pm 88^{* \#}$ \\
\hline
\end{tabular}

Results are expressed as mean $\pm \mathrm{SE}$. Blood samples were collected under the non-fasting condition. $* P<0.05$; significantly different from each control lean strain. ${ }^{\#} P<0.05$; significantly different between FLS-Lep $p^{o b} / L e p^{o b}$ and C57BL/6JWakShi- $L e p^{o b} /$ Lep $^{o b}$ mice. 
respectively. In females, both $L e p^{o b} / L e p^{o b}$ strains showed elevation of ALT activity.

\section{Liver lipid contents}

Liver wet weight and liver lipid contents in FLSLep ${ }^{o b} /$ Lep $^{o b}$, B6-Lep ${ }^{o b} / L_{e p} p^{o b}$, FLS, and C57BL/6JShi mice at 10 weeks of age are shown in Table 2. Male and female FLS-Lep ${ }^{o b} / L e p^{o b}$ mice showed significantly high liver wet weights and ratios of liver weight to body weight, compared with B6-Lep $p^{o b} / L e p^{o b}$ mice. In males, the triglyceride content in livers of FLS-Lep ${ }^{o b} / L e p^{o b}$ mice was $154.3 \mathrm{mg} / \mathrm{g}$ tissue, which was significantly higher (1.4-fold) than in liver of B6-Lep $p^{o b} /$ Lep $^{o b}$ mice. Liver cholesterol of male FLS-Lep $p^{o b} / L_{e} p^{o b}$ mice was also significantly higher (1.5-fold) than that of B6-Lep ${ }^{o b} / L^{2} p^{o b}$ mice. However, a difference in liver phospholipid content between FLS-Lep $p^{o b} / L e p^{o b}$ and B6-Lep $p^{o b} / L e p^{o b}$ mice was not found. In females, the triglyceride content of livers of FLS-Lep ${ }^{o b} / L e p^{o b}$ mice was 1.5-fold higher than that of livers of B6-Lep ${ }^{o b} / L e p^{o b}$ mice. Liver cholesterol of FLS-Lep $p^{o b} / L e p^{o b}$ mice was also significantly higher (1.4-fold) than that of B6-Lep ${ }^{o b} / \mathrm{Lep}^{o b}$ mice. Liver phospholipid was slightly but significantly higher in B6$L e p^{o b} / L e p^{o b}$ mice than in FLS-Lep $p^{o b} / L e p^{o b}$ mice. In lean mice, triglyceride and cholesterol contents of liver of FLS mice were significantly higher than those of C57BL/6JShi mice of both sexes.

\section{Pathological examination of the liver}

The liver of 10-week-old FLS-Lep ${ }^{o b} / L^{2} p^{o b}$ mice was remarkably enlarged and showed a whitish color (Fig. $5 \mathrm{~A})$. Histological examination also indicated that FLS$L e p^{o b} / L e p^{o b}$ mice had severe hepatic steatosis. The liver showed microvesicular steatosis and deposition of large lipid droplets in hepatocytes throughout the lobules (Fig. 5B). The hepatocytes showed remarkable hypertrophy and the nuclear displacement to the periphery of cells. Multifocal mononuclear cell infiltration was observed in the hepatic parenchyma of FLS-Lep $p^{o b} / L e p^{o b}$ mice at 20 weeks of age (Fig. 5C). Furthermore, livers of FLS$L e p^{o b} / L e p^{o b}$ mice contained multiple clusters of foamy macrophages, and showed mononuclear cell infiltration and necrosis with aging (Fig. 5D). Forty-week-old FLS mice livers had large lipid droplets, clusters of foamy cells, and monocyte infiltration (Fig. 5E). On the other hand, livers of B6-Lep ${ }^{o b} /$ Lep $^{o b}$ mice showed microvesicular steatosis with deposition of large lipid droplets around the central vein, but clusters of foamy cells and inflammation were not observed (Fig. 5F).

The incidence of hepatic tumors was examined for male and female FLS-Lep ${ }^{o b} / L e p^{o b}$ mice at 12 months of age. Whitish neoplastic nodules and reddish soft nodules were macroscopically found in $40 \%(8 / 20)$ and $20 \%$ $(4 / 20)$ of males, respectively. Neoplastic nodules were also found in $15 \%(3 / 20)$ of female FLS-Lep ${ }^{o b} /$ Lep $^{o b}$ mice and soft nodules in 5\% (1/20) at this age. Multiple nodules developed in many cases (Fig. 6A). The whitish

Table 2. Liver lipid contents in male and female FLS-Lep ${ }^{o b} / L e p^{o b}$, C57BL/6JWakShi-Lep ${ }^{o b} / L e p^{o b}$, FLS, and C57BL/6JShi mice at 10 weeks of age

\begin{tabular}{|c|c|c|c|c|c|c|c|c|}
\hline Sex & Strains & $\mathrm{n}$ & $\begin{array}{l}\text { Body wt. } \\
\text { (g) }\end{array}$ & $\begin{array}{l}\text { Liver wt. } \quad \mathrm{P} \\
(\mathrm{g})\end{array}$ & $\begin{array}{c}\text { Proportion of liver } \\
\text { (mg/g body wt.) }\end{array}$ & $\begin{array}{l}\text { Cholesterol } \\
\text { (mg/g tissue) }\end{array}$ & $\begin{array}{l}\text { Triglyceride } \\
\text { (mg/g tissue) }\end{array}$ & $\begin{array}{l}\text { Phospholipid } \\
\text { (mg/g tissue) }\end{array}$ \\
\hline \multicolumn{9}{|c|}{ Male } \\
\hline & 57BL/6JShi & 9 & $24.9 \pm 0.7$ & $1.21 \pm 0.07$ & $48.2 \pm 2.0$ & $3.0 \pm 0.2$ & $5.4 \pm 1.2$ & $20.9 \pm 0.5$ \\
\hline & LS & 8 & $32.2 \pm 0.4$ & $1.81 \pm 0.03$ & $56.1 \pm 0.7$ & $10.7 \pm 2.3$ & $38.9 \pm 11.2$ & $25.8 \pm 0.4$ \\
\hline & 57BL/6JWakShi-Lep ${ }^{o b} /$ Lep $^{o b}$ & 10 & $41.8 \pm 0.8^{*}$ & $4.06 \pm 0.13 *$ & $95.8 \pm 2.0^{*}$ & $23.2 \pm 1.9 *$ & $110.9 \pm 8.9 *$ & $22.6 \pm 0.5^{*}$ \\
\hline & LS-Lep $p^{o b} / L e p^{o b}$ & 8 & $43.2 \pm 0.5^{*}$ & $5.71 \pm 0.17$ *\# $^{\#}$ & $132.0 \pm 2.9^{* \#}$ & $34.6 \pm 2.1$ *\# & $154.3 \pm 8.1^{* \#}$ & $23.2 \pm 1.1^{*}$ \\
\hline \multicolumn{9}{|c|}{ Female } \\
\hline & $57 \mathrm{BL} / 6 \mathrm{JShi}$ & 10 & $21.5 \pm 0.2$ & $1.07 \pm 0.02$ & $49.9 \pm 0.7$ & $3.5 \pm 0.2$ & $5.6 \pm 1.5$ & $19.7 \pm 0.9$ \\
\hline & LS & 9 & $25.7 \pm 0.3$ & $1.25 \pm 0.02$ & $48.5 \pm 0.6$ & $5.2 \pm 0.5$ & $12.9 \pm 2.4$ & $21.5 \pm 1.4$ \\
\hline & 57BL/6JWakShi-Lep ${ }^{o b} /$ Lep $^{o b}$ & 10 & $43.5 \pm 0.9^{*}$ & $4.08 \pm 0.14 *$ & $93.6 \pm 1.7 *$ & $25.3 \pm 1.8^{*}$ & $125.0 \pm 6.2 *$ & $22.7 \pm 0.6^{*}$ \\
\hline & LS-Lep $p^{o b} / L_{e p}^{o b}$ & 8 & $43.6 \pm 0.8^{*}$ & $5.70 \pm 0.21{ }^{* \#}$ & $130.4 \pm 3.5^{* \#}$ & $36.2 \pm 1.4 * \#$ & $184.9 \pm 6.8^{* \#}$ & $20.9 \pm 0.6^{\#}$ \\
\hline
\end{tabular}

Results are expressed as mean $\pm \mathrm{SE}$. $* P<0.05$; significantly different from each control lean strain. ${ }^{\#} P<0.05$; significantly different between FLS-Lep ${ }^{o b} / L_{e p} p^{o b}$ and C57BL/6J/Shi-Lep $p^{o b} /$ Lep $^{o b}$ mice. 



Fig. 5. (A) The appearance of the liver of 10 -week-old FLS-Lep $p^{o b} / L e p^{o b}$ mice: enlargement and whitish in color. Light microscopic appearance of livers of the FLS-Lep $p^{o b} /$ Lep $^{o b}$ mice (B-D). (B) The liver of a 10-week-old female mouse showed microvesicular steatosis and deposition of large lipid droplets in hepatocytes throughout the lobules. (C) A liver section of a 20-week-old female mouse with diffuse large lipid droplets, and infiltration by mononuclear cells (arrows). (D) A liver section of a 40-week-old female mouse with clusters of foamy cells (arrows). (E) The liver of a 40-week-old female FLS mouse with large lipid droplets deposition, infiltration by mononuclear cells (asterisk), and clusters of foamy cells (arrows). (F) The liver of a 40-week-old female C57BL/6JWakShi-Lep ${ }^{o b} /$ Lep $^{o b}$ mouse showing microvesicular steatosis and deposition of large lipid droplets around the central vein, but without inflammation. C: central vein. P: portal vein. Hematoxylin-eosin staining (B-F). Bar $=100 \mu \mathrm{m}$.

nodule was histologically classified as a hepatocellular adenoma (Fig. 6B). The hepatocellular adenomas consisted of larger hepatocytes with round nuclei, which compressed adjacent hepatocytes. The adenoma cells had eosinophilic cytoplasm and nuclei of relatively constant size. The reddish cysts had the histological character of peliosis hepatis (Fig. 6C). Peliosis hepatis shows a disrupted parenchymal structure in which a thin column of hepatocytes is sandwiched by dilated sinusoidal spaces lined by prominent endothelial cells. Hepatocellular carcinomas were observed in 18-month-old FLS$L e p^{o b} / L e p^{o b}$ mice. The hepatocellular carcinomas were a well-differentiated type with a trabecular structure of tumorous hepatocytes (Fig. 6D). Hepatocellular carcinomas were occasionally found in adenomas (Fig. 6E). The deposition of large lipid droplets tended to decrease 

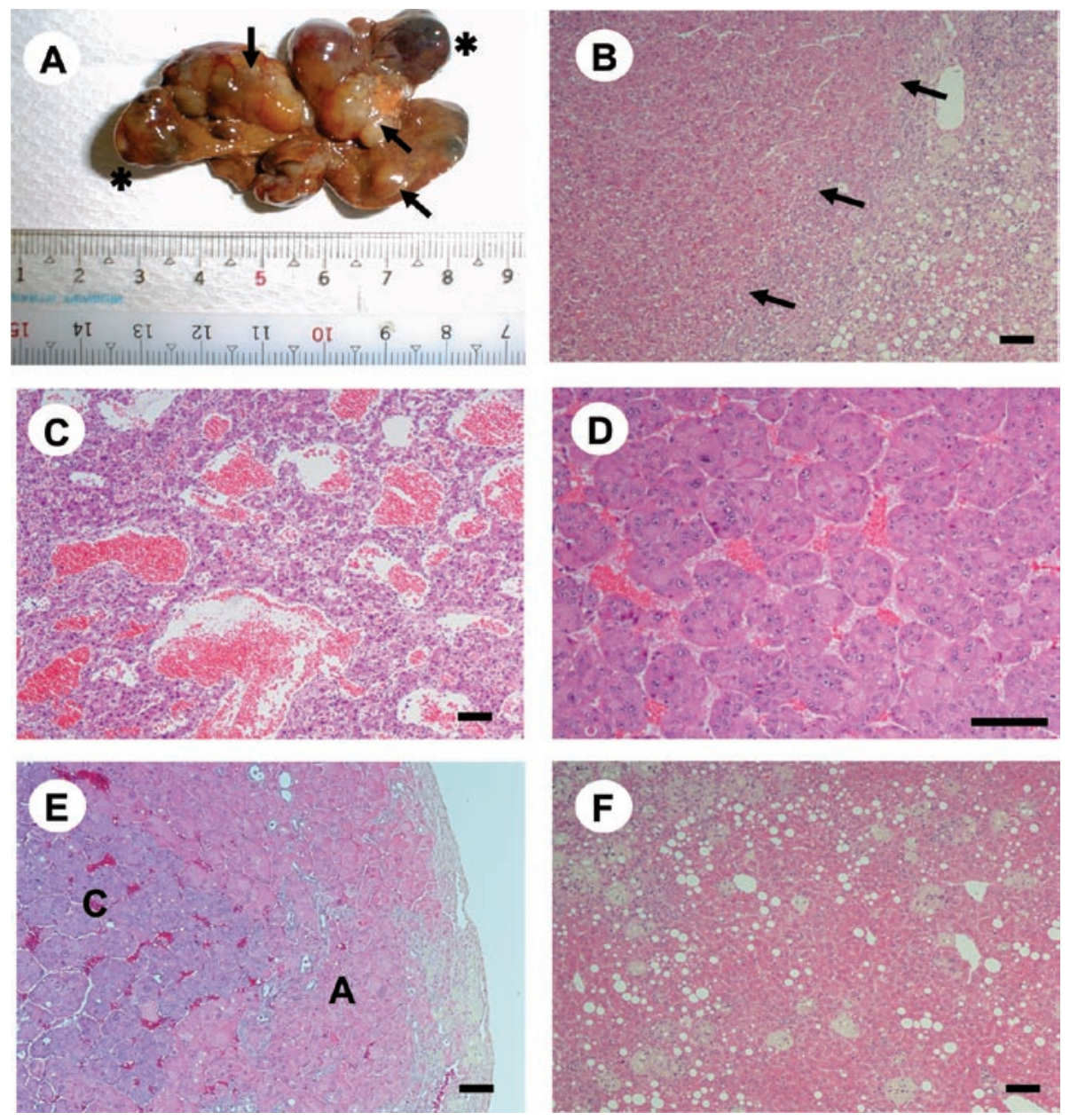

Fig. 6. Histological changes in livers of FLS-Lep $p^{o b} / L e p^{o b}$ mice. (A) The gross appearance of a liver with multiple nodules from a 17-month-old mouse. Arrows indicate whitish nodules with hepatocellular tumors. Asterisks indicate reddish nodules with peliosis hepatis. Light microscope appearance of livers from an 18-momth-old mice stained with hematoxylineosin (B-F). (B) Hepatocellular adenoma with distinctive border (arrows) between tumors and non-tumor parenchyma. (C) Peliosis hepatis with dilated sinusoidal space filled with numerous numbers of red blood cells. (D) Well-differentiated type of hepatocellular carcinoma with trabecular structure. (E) The hepatocellular carcinomas occasionally occurred in the center of adenomas. A: hepatocellular adenoma. C: hepatocellular carcinoma. (F) Lipid deposition and clusters of foamy cells in non-tumor area. Fatty change tended to decrease with aging. Bar $=100 \mu \mathrm{m}$.

with aging (Fig. 6F). The B6-Lep $p^{o b} / L e p^{o b}$ mice showed no evidence of hepatic tumors.

\section{Discussion}

Increase of obesity is an important human health problem in developed countries [26]. In the obese condition, the increasing insulin requirement of peripheral tissues due to excessive accumulation of body fat frequently progresses to insulin resistance. Fatty liver is observed in most obesity and type 2 diabetes mellitus patients, but its treatment has been neglected because it was thought to be a benign disease. In 1980, Ludwig et al. reported cases of steatohepatitis without excessive alcohol intake, and this form of steatohepatitis named non-alcoholic steatohepatitis (NASH). This finding indicated the possibility that fatty liver progresses to the hepatitis. Recently, hepatic steatosis without chronic alcohol intake 
and a clear cause has been called non-alcoholic fatty liver disease (NAFLD) [5]. NAFLD includes various conditions such as simple steatosis, NASH, and hepatic fibrosis, which is characterized by the presence of hepatic fatty change. NASH is closely related to obesity and insulin resistance. It was reported that the moderate fatty liver, NASH, and cirrhosis were found in 100, 50, and $19 \%$ of the severe obese patients with diabetes mellitus, respectively [29]. Only a few patients with NAFLD eventually develop more severe liver diseases cirrhosis and hepatocellular carcinoma, but the risk increases in the presence of NASH. Recent study have reported that many cases progress to liver cancer from NASH $[10,13,28]$. NASH has become an important health problem due to the increase of obesity and type 2 diabetes mellitus in developed countries.

We previously reported the establishment of FLS mouse [30]. The FLS mouse shows lipid (mainly triglyceride) accumulation in the liver from a young age. The results of a genetic cross study suggest that fatty liver formation in FLS mice is a complex polygenic trait [30]. Takahashi et al. suggested that apo C-III is a candidate causative factor of hypertriglycemia in FLS mice [32]. In addition to the lipid metabolism abnormalities in the liver and blood, steatohepatitis-like lesions including micro-abscesses and infiltration of mononuclear cells were observed in the liver of FLS mice. Interestingly, aged FLS mice frequently developed hepatic tumors including hepatocellular adenoma, carcinoma, and peliosis hepatis [31]. These findings suggest that the FLS mouse has potential as a model of steatohepatitis and hepato-carcinogenesis in human NASH. The relationship between NASH and carcinogenesis in FLS mice is not yet clear. However, Iwai et al. reported that FLS mice have the higher sensitivity to diethylnitrosamineinduced hepatocarcinogenesis than $\mathrm{C} 3 \mathrm{H}$ and $\mathrm{C} 57 \mathrm{BL}$ mice [14]. This finding suggests that FLS mice are susceptible to hepatic cancer by stimulation such as inflammation and DNA damage.

In the present study, we attempted to transfer the $L e p^{o b}$ gene into the FLS mouse to examine the influence of obesity on the hereditary fatty liver phenotype of the FLS mouse. A new congenic strain, the FLS-Lep $p^{o b}$ / $L e p^{o b}$ mouse, was established by backcross mating of FLS and B6-Lep $p^{o b} /+$ mice. Because FLS-Lep $p^{o b} / L e p^{o b}$ mice are infertile, $L e p^{o b}$ homozygous mice must be obtained by mating of $L e p^{o b}$ heterozygotes. In present, the identification of $L e p^{o b} /+$ mice for the mating was performed by the PCR restriction fragment length polymorphism method [11].

The nonsense mutation of the $L e p^{o b}$ gene for the mouse leptin protein accounts for the fatty phenotype in B6$L e p^{o b} / L^{o p} p^{o b}$ mice through impaired appetite control [7]. The leptin secretes from white adipose tissue with increase of the body fat, and suppresses feeding behavior and the increase of energy consumption through leptin receptors in the hypothalamus. FLS-Lep $p^{o b} / L e p^{o b}$ mice also showed early-onset obesity that was similar to that of B6-Lep ${ }^{o b} / \mathrm{Lep}^{o b}$ mice, and food consumption in FLS$L e p^{o b} / L e p^{o b}$ mice increased more than in B6-Lep $p^{o b} /$ $L e p^{o b}$ mice. Leptin mRNA overexpression was observed in FLS-Lep ${ }^{o b} /$ Lep $^{o b}$ mice, suggesting that the regulation of appetite is not controlled by the immature leptin protein.

The livers of 10-week-old FLS-Lep ${ }^{o b} /$ Lep $^{o b}$ mice were remarkably enlarged compared to those of B6-Lep ${ }^{o b} /$ $L e p^{o b}$ and FLS mice. The lipid contents such as triglyceride, cholesterol and phospholipid were very high in the FLS-Lep $p^{o b} / L e p^{o b}$ mice. The results of the histological examination also indicated that FLS-Lep $p^{o b} / L e p^{o b}$ mice have severe hepatic steatosis compare with other mouse strains.

Interestingly, FLS-Lep $p^{o b} / L e p^{o b}$ mice developed not only remarkable obesity due to the $L e p^{o b}$ mutation but also severe diabetic symptoms. The excretion of urinary glucose was already present in FLS-Lep $p^{o b} / L e p^{o b}$ mice at 3 weeks of age, and the incidence of overt diabetes reached $96-100 \%$ at $9-12$ weeks of age. The incidence of overt diabetes was extremely high compared with B6-Lep ${ }^{o b} / L^{2} p^{o b}$ mice. The difference of the water intake and urine excretion between FLS-Lep ${ }^{o b} / L_{e p}^{o b}$ and B6$L e p^{o b} / L e p^{o b}$ mice reflected the severity of diabetes well. At 9-12 weeks of age, FLS-Lep ${ }^{o b} / L e p^{o b}$ mice had severe insulin resistance manifested as hyperglycemia, hyperinsulinemia, and glucose intolerance. Since the backcrossing was performed without any selection for hyperglycemia or glucose intolerance, the development of severe diabetic symptoms in the FLS-Lep ${ }^{o b} / L^{o p} p^{o b}$ mice probably resulted from interaction of the genetic background of FLS mice and obesity due to the $L e p^{o b}$ gene 
mutation. These characters suggest that FLS-Lep ${ }^{o b} /$ $L e p^{o b}$ mice with hepatic steatosis and insulin resistance closely resemble NAFLD in humans. The results of OGTT also suggest that the FLS mouse has mild insulin resistance. Recently, Oze-Fukai et al. reported that FLS mice showed an age-dependent increase in glucose intolerance up to the age of 24 weeks. After that age, the glucose tolerance of the FLS mice spontaneously ameliorated and diabetes associated with hyperinsulinemia was resolved by 48 weeks of age. Islets of FLS mice demonstrated a marked increase in $\beta$-cell mass with an increase in $\beta$-cell numbers [23]. We have not yet examined the diabetic symptoms of the aged FLS-Lep ${ }^{o b} /$ $L e p^{o b}$ mice in detail, but the phenomenon like FLS mice may be a cause for the decrease in overt diabetes seen in FLS-Lep ${ }^{o b} / L e p^{o b}$ mice with aging.

The histological features of human NASH are steatosis, ballooning degeneration of hepatocytes, Mallory bodies, hepatic inflammation, and hepatic pericellular fibrosis [18]. The hepatocytes including fat in FLS-Lep $p^{o b} /$ Lep $^{o b}$ and FLS mice enlarged remarkably, and we regarded them as a ballooning degeneration of the hepatocytes. Remarkably enlarged hepatocytes were not observed in the livers of the B6-Lep $p^{o b} / L e p^{o b}$ mice. The FLS-Lep $p^{o b} /$ $L e p^{o b}$ mice developed steatohepatitis-like lesions including mononuclear cell infiltration, clusters of foamy macrophages, and necrosis following hepatic steatosis. These inflammatory reactions were observed throughout the lobules, and their degree was stronger in FLS-Lep ${ }^{o b}$ / $L e p^{o b}$ mice than in FLS mice. In addition, slight fiber deposition was observed in the sinusoids of the FLS mice in preliminary examinations using azan staining. Recently, Kawahara et al. reported the existence of pericellular fibrosis in the livers of FLS-Lep ${ }^{o b} / L e p^{o b}$ mice [16]. We have not yet examined the hepatic fibrosis of FLS$L e p^{o b} / L^{o p} p^{o b}$ mice in detail. The progression to steatohepatitis from hepatic steatosis has not yet been elucidated but the two-hit theory has been widely accepted as an explanation of the development of NASH [4]. High calorie intake, excessive influx of free fatty acids into liver from adipose tissue and insufficient hepatic lipid secretion are considered to be causes of steatosis, and these conditions are related to insulin resistance [33]. The continuous accumulation of lipids in the liver is the first hit and it makes the liver vulnerable to the second hit. The second hit consists of increased oxidative stress (lipid peroxidation, decreased hepatic ATP production, and induction of pro-inflammatory cytokines), which leads to hepatic inflammation and fibrosis. Iwai et al. reported that the level of 8-hydroxy-2'-deoxyguanosine (8-OHdG), a marker of oxidative DNA damage, increased more in the livers of FLS mice than in $\mathrm{C} 3 \mathrm{H}$ and C57BL/6N mice [14]. Kawahara et al. reported that increase of oxidative stress marker as $8-\mathrm{OHdG}$ and 4-hydroxy-nonenal, was detected in the liver of FLS$L e p^{o b} / L^{o p} p^{o b}$ mice [16]. Fenofibrate (a peroxisome proliferator-activated receptor $\alpha$ agonist) improved hepatic steatosis and lipid peroxidation in FLS mice [8]. These findings suggest that hepatic oxidative stress plays an important role in the development of steatohepatitis in FLS and FLS-Lep ${ }^{o b} /$ Lep $^{o b}$ mice.

FLS mice develop hepatic tumors following steatohepatitis. The incidence of hepatic tumors in male FLS mice was $9 \%$ at 13-14 months of age [31]. The FLS$L e p^{o b} / L^{o p}{ }^{o b}$ mice also developed hepatic tumors, and their incidence was high compared with FLS mice at 12 months of age. The hepatic tumors were multiple in many cases. The whitish nodules were histologically classified as hepatocellular adenoma and/or carcinoma. Hepatocellular carcinomas were occasionally found in the center area of adenomas with aging. The hepatocellular carcinomas were a well-differentiated type with a trabecular structure of tumorous hepatocytes. In a preliminary study, 18-month-old male and female FLS$L e p^{o b} / L^{o p} p^{o b}$ mice had hepatic tumors with an incidence of $100 \%$, a higher incidence than FLS mice $(93 \%$ in males and $0 \%$ in females). Fat deposition in the nontumor area of livers of FLS-Lep ${ }^{o b} / L e p^{o b}$ and FLS mice with hepatic tumors tended to decrease. This finding resembles "burn-out NASH" in humans, in which fat deposition decreases at the time of the development of cirrhosis and hepatic cancer [24]. In humans, the development to the liver cancer from fatty liver has attracted attention. It is thought that the accumulation of DNA damage under chronic hepatic inflammation, as in NASH, induces carcinogenesis. The differences in tumor prevalence in FLS- $L e p^{o b} / L e p^{o b}$ and FLS mice may be related to the degree of the fatty change and inflammation in the liver. Hepatitis $\mathrm{C}$ virus (HCV) infection is a major cause of chronic hepatitis, which eventually leads to hepatic 
carcinogenesis. Steatosis of the liver is a histological features of hepatitis $C$ [1]. The transgenic expression of $\mathrm{HCV}$ core protein in mice has been reported to induce both hepatic steatosis and HCC, although inflammation in the liver was not observed in transgenic mice [19, 21]. Although the HCV core protein transgenic mice did not have inflammation, it was shown that production of reactive oxygen species (ROS) increased [20]. These findings suggest that chronic steatosis in the liver is also a risk for hepatocarcinogenesis, and the risk increases in the presence of chronic inflammation such as NASH.

Recently, Horie et al. reported that the hepatocytespecific phosphatase and tensin homolog (PTEN) deficient mouse develops steatohepatitis and hepatocellular carcinoma. [12]. However, this mouse does not show obesity and insulin resistance, and has characteristics which are different from NASH in humans.

In conclusion, the FLS-Lep ${ }^{o b} / L e p^{o b}$ mice developed steatohepatitis and hepatic tumors following hepatic steatosis. The path from hepatic steatosis to hepatocarcinogenesis in the FLS-Lep ${ }^{o b} / L e p^{o b}$ mice is not clear. However, the FLS-Lep ${ }^{o b} / L_{e p} p^{o b}$ mouse with obesity and type 2 diabetes mellitus might be a useful animal model for human NASH. Further studies may clarify the relationship between NASH and metabolic disorders such as insulin resistance and obesity.

\section{Acknowledgments}

We would like to thank Tadao Oohara, Kiyoshi Tsukahara, Mitsuyoshi Fijiwara, and Hisae Saji for management and care of mice, Yukiko Nakai for assistance with biochemical analysis, and Dr. Kenji Kato and Dr. Reiji Takeda for their continuous support and encouragement.

\section{References}

1. Bach, N., Thung, S.N., and Schaffner, F. 1992. The histological features of chronic hepatitis $\mathrm{C}$ and autoimmune chronic hepatitis: a comparative analysis. Hepatology 15: 572-577.

2. Bugianesi, E., Leone, N., Vanni, E., Marchesini, G., Brunello, F., Carcucci, P., Musso, A., De Paolis, P., Capussotti, L., Salizzoni, M., and Rizzetto, M. 2002. Expanding the natural history of non-alcoholic steatohepatitis: from cryptogenic cirrhosis to hepatocellular carcinoma. Gastroenterology 123:
134-140.

3. Chittturi, S.A., Abeygunasekera, S., Farrell, G.C., HolmesWalker, J., Hui, J.M., Fung, C., Karim, R., Lin, R., Samarasinghe, D., Liddle, C., Weltman, M., and George, J. 2002. NASH and insulin resistance: insulin hypersecretion and specific association with the insulin resistance syndrome. Hepatology 35: 373-379.

4. Day, C.P. and James, O.F. 1998. Steatohepatitis: a tale of two 'hit'? Gastroenterology 114: 842-845.

5. Diehl,A.M. 1999. Nonalcoholic steatohepatitis. Semin.Liver Dis. 16: 221-229.

6. Folch, J., Lees, M., and Sloane Stanley, G.H. 1957. A simple method for the isolation and purification of total lipids from animal tissues. J. Biol. Chem. 226: 497-509.

7. Halaas, J.L., Gajiwala, K.S., Maffei, M., Cohen, S.L., Chait, B.T., Rabinowitz, D., Lallone, R., Burley, S.K., and Friedman, J.M. 1995. Weight-reducing effects of the plasma protein encoded by the obese gene. Science 269: 543-546.

8. Harano, Y., Yasui, K., Toyama, T., Nakajima, T., Mitsuyoshi, H., Minami, M., Hirasawa, T., Itoh, Y., and Okanoue, T. 2006. Fenofibrate, a peroxisome proliferator-activated receptor alpha agonist, reduces hepatic steatosis and lipid peroxidation in fatty liver shionogi mice with hereditary fatty liver. Liver Int. 26: 613-620.

9. Hashimoto, E., Yatsuji, S., Kaneda, H., Yoshioka, Y., Taniai, M., Tokushige, K., and Shiratori, K. 2005. The characteristics and natural history of Japanese patients with nonalcoholic fatty liver disease. Hepatol. Res. 33: 72-76.

10. Hashizume, H., Sato, K., Takagi, H., Hirokawa, T., Kojima, A., Sohara, N., Kakizaki, S., Mochida, Y., Shimura, T., Sunose, Y., Ohwada, S., and Mori, M. 2007. Primary liver cancers with nonalcoholic steatohepatitis. Eur. J. Gastroenterol. Hepatol. 19: 827-834.

11. Hirasawa, T., Ohara, T., and Makino, S. 1997. Genetic typing of the mouse $o b$ mutation by PCR and restriction enzyme analysis. Exp. Anim. 46: 75-78.

12. Horie, Y., Suzuki, A., Kataoka, E., Sasaki, T., Hamada, K., Sasaki, J., Mizuno, K., Hasegawa, G., Kishimoto, H., Iizuka, M., Naito, M., Enomoto, K., Watanabe, S., Mak, T. W., and Nakano, T. 2004. Hepatocyte-specific Pten deficiency results in steatohepatitis and hepatocellular carcinomas. J. Clin. Invest. 113: 1774-1783.

13. Ichikawa, T., Yanagi, K., Motoyoshi, Y., Hamasaki, K., Nakao, K., Toriyama, K., and Eguchi, K. 2006. Two cases of non-alcoholic steatohepatitis with development of hepatocellular carcinomas without cirrhosis. J. Gastroenterol. Hepatol. 21: 1865-1866.

14. Iwai, S., Murai, T., Makino, S., Min, W., Morimura, K., Mori, S., Hagihara, A., Seki, S., and Fukushima, S. 2007. High sensitivity of fatty liver shionogi (FLS) mice to diethylnitrosamine hepatocarcinogenesis: comparison to C3H and C57 mice. Cancer Lett. 246: 115-121.

15. Japanese Association for Laboratory Animal Science. 1987. Guidelines for animal experimentation. Jikken Dobutsu 36: 285-288.

16. Kawahara, H. and Takase, S. 2006. Mutation of mitochondrial DNA in patients with nonalcoholic steatohepatitis. Nippon Rinsho 64: 1095-1099 (in Japanese). 
17. Ludwig, J., Vigianon, T.R., McGill, D.B., and Oh, B.J. 1980. Non-alcoholic steatohepatitis: Mayo Clinic experience with a hitherto unnamed disease. Mayo Clin. Proc. 55: 434438.

18. Matteoni, C.A., Younossi, Z.M., Gramlich, T., Boparai, N., Liu, Y.C., and McCullough, A.J. 1999. Nonalcoholic fatty liver disease: a spectrum of clinical and pathological severity. Gastroenterology 116: 1413-1419.

19. Moriya, K., Fujie, H., Shintani, Y., Yotsuyanagi, H., Tsutsumi, T., Ishibashi, K., Matsuura, Y., Kimura, S., Miyamura, T., and Koike, K. The core protein of hepatitis $\mathrm{C}$ virus induces hepatocellular carcinoma in transgenic mice. 1998. Nat. Med. 4: 1065-1067.

20. Moriya, K., Nakagawa, K., Santa, T., Shintani, Y., Fujie, H., Miyoshi, H., Tsutsumi, T., Miyazawa, T., Ishibashi, K., Horie, T., Imai, K., Todoroki, T., Kimura, S., and Koike, K. 2001. Oxdative stress in the absence of inflammation in a mouse model for hepatitis $\mathrm{C}$ virus-associated hepatocarcinogenesis. Cancer Res. 61: 4365-4370.

21. Moriya, K., Yotsuyanagi, H., Shintani, Y., Fujie, H., Ishibashi, K., Matsuura, Y., Miyamura, T., and Koike, K. 1997. Hepatitis C virus core protein induces hepatic steatosis in transgenic mice. J. Gen. Virol. 78: 1527-1531.

22. Neuschwander Tetri, B.A. and Caldwell, S.H. 2003. Nonalcoholic steatohepatitis: summary of an AASLD Single Topic Conference. Hepatology 37: 1202-1219.

23. Oze-Fukai, A., Fuzisawa, T., Sugimoto, K., Nojima, K., Shindo, N., Shimoyoshi, S., Yoshikawa, Y., Sato, Y., Shimomura, I., Ikegami, H., and Rakugi, H. 2009. A novel mouse model for type 2 diabetes and non-alcoholic fatty liver disease: spontaneous amelioration of diabetes by augmented beta cell mass. Endocr. J. 56: 227-234.

24. Poonawala, A., Nair, S.P., and Thuluvath, P.J. 2000. Prevalence of obesity and diabetes in patients with cryptogenic cirrhosis: a case-control study. Hepatology 32 : 689-692.

25. Powell, E.E., Cooksley, W.G., Hanson, R., Searle, J., Halliday, J.W., and Powell, L.W. 1990. The natural history of nonalcoholic steatohepatitis: a follow-up study of fortytwo patients for up to 21 years. Hepatology 11: 74-80.
26. Rector, R.S., Thyfault, J.P., Wei, Y., and Ibdah, J.A. 2008. Non-alcoholic fatty liver disease and the metabolic syndrome: an update. World J. Gastroenterol. 14: 185192.

27. Reid,A.E. 2001. Nonalcoholic steatoheptitis. Gastroenterology 121: 710-723.

28. Sanyal, A.J., Banas, C., Sargeant, C., Luketic, V.A., Sterling, R.K., Stravitz, R.T., Shiffman, M.L., Heuman, D., Coterrell, A., Fisher, R.A., Contos, M.J., and Mills, A.S. 2006. Similarities and differences in outcomes of cirrhosis due to non-alcoholic steatohepatitis and hepatic C. Hepatology 43: 682-689.

29. Silverman, J.F., Pories, W.J., and Caro, J.F. 1989. Liver pathology in diabetes mellitus and morbid obesity: clinical, pathological and biochemical considerations. Pathol.Annu. 24: 275-302.

30. Soga, M., Kishimoto, Y., Kawaguchi, J., Nakai, Y., Kawamura, Y., Inagaki, S., Katoh, K., Oohara, T., Makino, S., and Oshima, I. 1999. The FLS mouse: a new inbred strain with spontaneous fatty liver. Lab. Anim. Sci. 49: 269-275.

31. Soga, M., Kishimoto, Y., Kawamura, Y., Inagaki, S., Makino, S., and Saibara, T. 2003. Spontaneous development of hepatocellular carcinomas in the FLS mice with hereditary fatty liver. Cancer Lett. 196: 43-48.

32. Takahashi, M., Saibara, T., Nemoto, Y., Ono, M., Akisawa, N., Iwasaki, S., Toda, K., Ogawa, Y., Wakatsuki, A., Inagaki, S., and Onishi, S. 2003. A novel type hypertriglycemia observed in FLS mice. Lipids 38: 687-692.

33. Utzschneider, K.M. and Kahn, S.E. 2006. Review: the role of insulin resistance in nonalcoholic fatty liver disease. $J$. Clin. Endocrinol. Metab. 91: 4753-4761.

34. Yatsuji, S., Hashimoto, E., Kaneda, H., Taniai, M., Takakura, M., Tokushige, K., and Shiratori, K. 2005. Characteristic features and risk factors for hepatocellular carcinoma in nonalcoholic steatohepatitis. Hepatology 42: 625A.

35. Zhang, Y., Proenca, R., Maffei, M., Barone, M., Leopold, L., and Friedman, J. M. 1994. Positional cloning of the mouse obese gene and its human homologue. Nature 372 : $425-432$. 